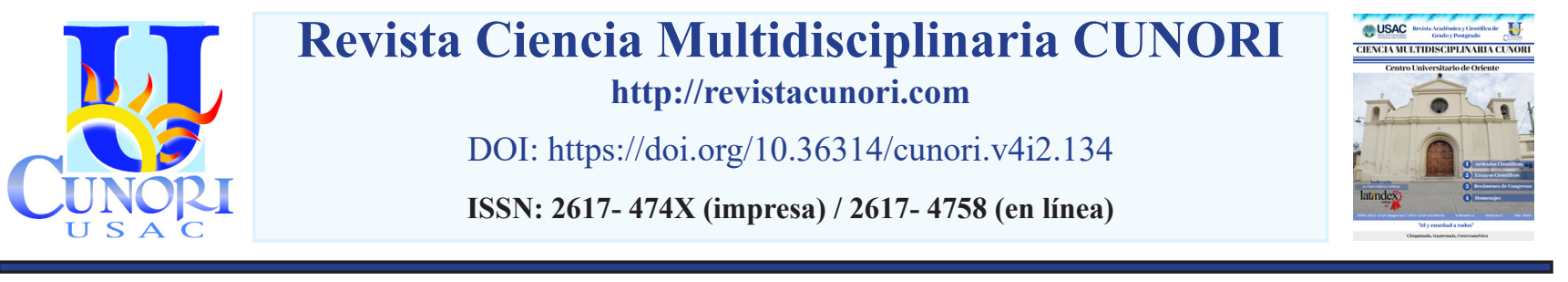

Referencia del artículo

Morales-Guzmán, R. R. (2020). La evaluación que practican los docentes universitarios. Revista Ciencia Multidisciplinaria Cunori, 4(2), 109-115. https://doi.org/10.36314/cunori.v4i2.134

\title{
La evaluación que practican los docentes universitarios
}

\section{The evaluation practiced by university teachers}

\author{
Rudy Rolando Morales Guzmán \\ Universidad de San Carlos de Guatemala \\ https://orcid.org/0000-0001-8338-967X
}

Recibido: 13 de septiembre de 2020 / Revisado: 14 de septiembre de 2020 / Aceptado: 10 de mayo de 2020

Disponible en internet el 30 de octubre de 2020

$\begin{array}{ll}\text { Resumen } & * \text { Autor para correspondencia. } \\ \text { Correo electrónico: permaneciendoencristo@gmail.com }\end{array}$

$\mathrm{F}$ recuentemente las evaluaciones han sido parámetro para la medición de conocimientos teóricos y en algunos casos sirven para abusar de la memorización de los estudiantes. Al respecto, Careaga (2001) declara que no es eventual que se evalúe más la competencia mental que la manual o que se les preste más atención a los aspectos intelectuales que a los del análisis. Por lo que fue de suma importancia analizar la evaluación que practican los docentes universitarios, para mejorar la calidad del aprendizaje. La metodología utilizada fue descriptiva e hipotética-deductiva. La muestra correspondió a 169 estudiantes de la carrera de Profesorado de Enseñanza Media en Pedagogía y Técnico en Administración Educativa del -CUNORI-. Considerando que la universidad está formando profesores que pueden replicar la práctica evaluativa en otros entornos de aprendizaje, se utilizó el modelo elaborado por De la Orden Hoz Arturo y Pimienta Prieto Julio (2016), en el cual se describen tres tipos de evaluación, los cuales son: la evaluación sumativa, formativa y para el aprendizaje. Se comprobó la hipótesis de investigación que planteaba "La evaluación que practican los docentes en la carrera de Profesorado de Enseñanza Media en Pedagogía y Técnico en Administración Educativa del Centro Universitario de Oriente, se encuentra en un nivel medio", ubicándose en un 61.5 por ciento, esto equivale a que, de 30 indicadores, 20 se encuentran arriba de la regularidad en su ejecución. De 21 docentes, 13 están aplicando pruebas objetivas y alternativas enfocándose en la calificación y el aprendizaje de sus estudiantes.

Palabras clave: aprendizaje, docente, evaluación

\section{Abstract}

Grequently, evaluations have been a parameter for measuring theoretical knowledge and in some cases they serve to abuse - students' memorization. In this regard, Careaga (2001) states that it is not possible that mental competence is evaluated more than manual competence or that more attention is paid to intellectual aspects than to those of analysis. Therefore, it was of utmost importance to analyze the evaluation carried out by university teachers, to improve the quality of learning. The methodology used was descriptive and hypothetical-deductive. The sample corresponded to 169 students of the career of Teaching Staff of Secondary Education in Pedagogy and Technician in Educational Administration of -CUNORI-. Considering that the university is training teachers who can replicate the evaluative practice in other learning environments, the model developed by De la Orden Hoz Arturo y Pimienta Prieto Julio (2016) was used, in which three types of evaluation are described, which They are: summative, formative and learning evaluation. The research hypothesis that posed was verified: "The evaluation practiced by teachers in the career of Teaching Staff of Secondary Education in Pedagogy and Technician in Educational Administration of the University Center of the 
East, is at an average level", ranking at 61.5 per percent, this is equivalent to that, of 30 indicators, 20 are above regularity in their execution. Of 21 teachers, 13 are taking objective and alternative tests focusing on the grading and learning of their students.

Keywords: learning, teaching, evaluation

\section{Introducción}

En la actualidad los procesos evaluativos deben estar acordes a las exigencias de un aprendizaje armónico e integral, las prácticas evaluativas no son ajenas a dichos procesos, están íntimamente vinculadas, y los instrumentos y técnicas no sólo pueden ser para medición de conocimientos teóricos. La práctica evaluativa en los procesos de aprendizaje implica la utilización de nuevas técnicas e instrumentos de evaluación y el cambio de paradigma del proceso, en donde el enfoque busque ser más formativo y la evaluación deje de perseguir solamente una nota o punteo. Es indispensable que los procesos de evaluación en la universidad sean modelo para los niveles inferiores para que cuando los estudiantes ingresen a la universidad no les sean ajenos dichos procesos evaluativos. Por las razones anteriores, fue de suma importancia analizar la evaluación que practican los docentes durante el proceso de aprendizaje La investigación fue indispensable para el aporte de un modelo evaluativo que reduzca los errores en las prácticas evaluativas docentes, haciendo uso de mecanismos de evaluación que incentivan completamente al aprendizaje en los estudiantes y que en muchos casos puede evitar el fracaso escolar.

Para dar ese salto de la educación tradicional al aprendizaje significativo, es importante innovar y adoptar nuevas formas de evaluación. Esto debido a que actualmente, las realidades educativas se caracterizan por ser dinámicas y flexibles, con una evolución vertiginosa del conocimiento y su difusión a gran escala y rapidez (Arias et al., 2011). De acuerdo a Ruano (2002), citado por Choc (2014) en el presente se debe reflexionar en la evaluación como un proceso que fomente la autoevaluación, coevaluación, reflexión y mejoramiento continuo. Se puede pensar también en que toda evaluación debe servir para contribuir con el rendimiento y la efectividad en el aprendizaje.

Los resultados del estudio realizado evidencian que los docentes están utilizando las pruebas como instrumentos de evaluación, pero también de aprendizaje, aunque necesitan mayor conocimiento y aplicación efectiva de las evaluaciones en línea. De igual manera que los docentes devuelven los trabajos con comentarios, pero precisan fortalecer los criterios de evaluación en función de las habilidades de los estudiantes. Así mismo, que los docentes enfocan la evaluación en el proceso, pues les interesan las cualidades de sus estudiantes, pero requieren fortalecer la coevaluación, autoevaluación y retroalimentación. Y también que los docentes están aplicando pruebas objetivas y alternativas, pero urge que fortalezcan las técnicas y estrategias cualitativas de evaluación y las evaluaciones de análisis y prácticas.

Diferentes investigaciones se han realizado sobre el tema de evaluación, sus procesos, prácticas y enfoques. Dentro de todo este gran campo de estudio se encuentran inmersos los tipos de evaluación, siendo predominante la evaluación sumativa y sus instrumentos de índole objetivo. El estudio analizado en la Tesis Doctoral Zambrano (2014) "Prácticas evaluativas para la mejora de la calidad del aprendizaje: Un estudio contextualizado en La Unión, Chile de la Universidad Autónoma de Barcelona, España”, confirma que se reflejan limitaciones en los procesos de evaluación, pues aún los docentes investigados 
manifestaron que utilizaron una mayor cantidad de pruebas escritas y exámenes tradicionales, no siendo concordante con la aproximación centrada en el proceso. Según tesis de maestría sobre la "Caracterización y análisis de las prácticas de evaluación, una mirada a la acción docente para la mejora de la enseñanza", Hernández (2015) la sensación sobre la evaluación de los aprendizajes influye en la escogencia de las estrategias y métodos con los que se estima a los estudiantes, lo que se relaciona con los resultados en la investigación de Puentes (2009) al concluir que se debe ver la práctica de la evaluación de los aprendizajes como una organización fundamentada en las tareas de aprendizaje y las pericias integrales que se pretende alcanzar en el estudiante, para lo cual, es preciso no solamente reconocer lo cognitivo, sino también los desempeños, lo afectivo y la aptitud. De acuerdo a la tesis Doctoral Monteagudo (2014) "Las prácticas de evaluación en la materia de historia de cuarto grado de ESO en la Comunidad Autónoma de la Región de Murcia", las evaluaciones teóricas y trabajos son las herramientas de apreciación del aprendizaje de los estudiantes más utilizadas. Según la tesis de maestría "El Proceso de Evaluación en el Aprendizaje de los estudiantes de la carrera de Profesorado de Enseñanza Media en Pedagogía y Ciencias de la Educación del Centro Universitario de San Marcos, de la Universidad de San Carlos de Guatemala", Velásquez (2017), se determinó que el proceso de evaluación que se aplica, centra su interés en las pruebas objetivas, en las calificaciones, en la aprobación de cursos y certificación de estudios.

El objeto de estudio de la investigación realizada fue la evaluación que practican los docentes, la cual busca obtener explicaciones sobre el funcionamiento del proceso enseñanza-aprendizaje para introducir eventualmente cambios para mejorarlo" (Acuña et al., 2010). Es un proceso complejo porque está vinculado con el quehacer docente en la planificación, las prácticas andragógicas, las actividades, las estrategias, las formas y los resultados para la toma de decisiones que pueden favorecer o perjudicar a los actores principales del sistema educativo.

\section{Materiales y métodos}

El método que se utilizó es el hipotético-deductivo. Las técnicas empleadas en el presente estudio y tomando en cuenta el tipo de investigación descriptiva, fueron la encuesta para estudiantes, entrevista para docentes, observación documental y análisis de registros. Para efectos de la recolección de la información se tomó una muestra probabilística estratificada de los estudiantes del primero, tercero, quinto y séptimo semestre, en donde, de 292 estudiantes participaron 169.

\section{Resultados}

Se presentan los resultados de la investigación realizada en la carrera de Profesorado de Enseñanza Media en Pedagogía y Técnico en Administración Educativa del Centro Universitario de Oriente. Todas las hipótesis específicas como la general fueron aceptadas, eso significa que tanto la evaluación sumativa, formativa, para el aprendizaje y evaluación docente, se están aplicando, de manera permisible. La subvariable evaluación para el aprendizaje es la que se encuentra con menos nivel de aplicación con un 50.3\%. Particular atención merece el quinto semestre, en donde $62.9 \%$ ubica la evaluación para el aprendizaje en el nivel medio, siendo este semestre el de mayor porcentaje. Por lo que se debe fortalecer la práctica de la evaluación para el aprendizaje a este nivel principalmente en los cursos que se desa- 
rrollan en ese semestre, los cuales son: Administración General I, Organización Escolar I, Contabilidad General, Seminario y Laboratorio de Formación Docente.

Tabla No. 1 Evaluación sumativa según el semestre de los estudiantes en la carrera de Profesorado de Enseñanza Media en Pedagogía y Técnico en Administración Educativa (Junio, 2020).

\begin{tabular}{|c|c|c|c|c|c|}
\hline \multirow[b]{2}{*}{$\begin{array}{c}\text { Nivel de Evaluación } \\
\text { Sumativa }\end{array}$} & \multicolumn{4}{|c|}{ Semestre } & \multirow[b]{2}{*}{ Total } \\
\hline & Primero & Tercero & Quinto & Séptimo & \\
\hline Muy bajo & 0 & 0 & 1 & 0 & 1 \\
\hline Bajo & $\begin{array}{c}0.0 \% \\
11\end{array}$ & $0.0 \%$ & $\begin{array}{l}2.9 \% \\
6\end{array}$ & $\begin{array}{l}0.0 \% \\
4\end{array}$ & $\begin{array}{l}0.6 \% \\
28\end{array}$ \\
\hline Medio & $\begin{array}{c}21.6 \% \\
26\end{array}$ & $17.9 \%$ & $\begin{array}{c}17.1 \% \\
18\end{array}$ & $\begin{array}{l}9.1 \% \\
29\end{array}$ & $\begin{array}{l}16.6 \% \\
90\end{array}$ \\
\hline \multirow[t]{2}{*}{ Alto } & $\begin{array}{c}51.0 \% \\
14\end{array}$ & $\begin{array}{l}43.6 \% \\
15\end{array}$ & $\begin{array}{c}51.4 \% \\
10\end{array}$ & 65.9\% & $\begin{array}{l}53.3 \% \\
50\end{array}$ \\
\hline & $27.5 \%$ & $38.5 \%$ & $28.6 \%$ & $25.0 \%$ & $29.6 \%$ \\
\hline \multirow[t]{2}{*}{ Total } & 51 & 39 & 35 & 44 & 169 \\
\hline & $100 \%$ & $100 \%$ & $100 \%$ & $100 \%$ & $100 \%$ \\
\hline
\end{tabular}

Morales-Guzmán (2020). La evaluación que practican los docentes universitarios.

En referencia a la tabla anterior, se pudo establecer que el 53.3\% de los docentes utilizan la evaluación sumativa en un nivel "medio", por lo tanto el nivel es aceptable, pero se puede mejorar, razón por la cual se acepta la hipótesis de investigación H1: La evaluación sumativa es aplicada por los docentes en un nivel "medio". Significa que en las actividades presenciales y virtuales, de 21 docentes, 11 aplican un modelo evaluativo tradicional. Utilizan pruebas objetivas, pero en ocasiones varían con pruebas novedosas y de igual manera las evaluaciones son instrumentos de calificación pero también de aprendizaje. Estos resultados son los esperados pues coinciden con la hipótesis previamente planteada.

Tabla No. 2 Evaluación formativa según el semestre de los estudiantes en la carrera de Profesorado de Enseñanza Media en Pedagogía y Técnico en Administración Educativa (Junio, 2020).

\begin{tabular}{|c|c|c|c|c|c|}
\hline \multirow[b]{2}{*}{$\begin{array}{c}\text { Nivel de Evaluación } \\
\text { Formativa }\end{array}$} & \multicolumn{4}{|c|}{ Semestre } & \multirow[b]{2}{*}{ Total } \\
\hline & Primero & Tercero & Quinto & Séptimo & \\
\hline Muy bajo & 2 & 1 & 1 & 0 & 4 \\
\hline Bajo & $\begin{array}{c}3.9 \% \\
14\end{array}$ & $\begin{array}{l}2.6 \% \\
10\end{array}$ & $\begin{array}{l}2.9 \% \\
13\end{array}$ & $\begin{array}{c}0.0 \% \\
11\end{array}$ & $\begin{array}{l}2.4 \% \\
48\end{array}$ \\
\hline Medio & $\begin{array}{c}27.5 \% \\
29\end{array}$ & $\begin{array}{l}25.6 \% \\
24\end{array}$ & $\begin{array}{c}37.1 \% \\
18\end{array}$ & $\begin{array}{l}25.0 \% \\
26\end{array}$ & $28.4 \%$ \\
\hline Alto & $\begin{array}{l}56.9 \% \\
6\end{array}$ & $\begin{array}{c}61.5 \% \\
4\end{array}$ & $\begin{array}{c}51.4 \% \\
3\end{array}$ & $\begin{array}{l}59.1 \% \\
7\end{array}$ & $\begin{array}{l}57.4 \% \\
20\end{array}$ \\
\hline Total & $\frac{11.8 \%}{51}$ & $\begin{array}{c}10.3 \% \\
39\end{array}$ & $\begin{array}{l}8.6 \% \\
35\end{array}$ & $\frac{15.9 \%}{44}$ & $\begin{array}{c}11.8 \% \\
169\end{array}$ \\
\hline & $100 \%$ & $100 \%$ & $100 \%$ & $100 \%$ & $100 \%$ \\
\hline
\end{tabular}

Morales-Guzmán (2020). La evaluación que practican los docentes universitarios. 
En referencia a la tabla anterior, se pudo establecer que el 57.4\% de los docentes utilizan la evaluación formativa en un nivel "medio", razón por la cual se acepta la hipótesis de investigación H2: La evaluación formativa es aplicada por los docentes en un nivel "medio". Significa que de 21 docentes, 12 comparan el resultado de la evaluación con criterios que previamente establecieron, por ejemplo, para una mesa redonda; establecen estos criterios: A) participación. B) Opiniones convincentes. C) Argumentación fundamentada en información bibliográfica. Estos resultados son los esperados, pues aciertan con la hipótesis de investigación.

Tabla No. 3 Evaluación para el aprendizaje según el semestre de los estudiantes en la carrera de Profesorado de Enseñanza Media en Pedagogía y Técnico en Administración Educativa (Junio, 2020).

\begin{tabular}{|c|c|c|c|c|c|}
\hline \multirow{3}{*}{$\begin{array}{l}\text { Nivel de Evaluación para } \\
\text { el Aprendizaje } \\
\text { Muy bajo }\end{array}$} & \multicolumn{4}{|c|}{ Semestre } & \multirow[b]{2}{*}{ Total } \\
\hline & Primero & Tercero & Quinto & Séptimo & \\
\hline & 1 & $\sigma$ & 2 & 0 & 3 \\
\hline Bajo & $\begin{array}{c}2.0 \% \\
13\end{array}$ & $\begin{array}{c}0.0 \% \\
9\end{array}$ & $\begin{array}{c}5.7 \% \\
7\end{array}$ & $\begin{array}{c}0.0 \% \\
8\end{array}$ & $\begin{array}{c}1.8 \% \\
37\end{array}$ \\
\hline Medio & $\begin{array}{c}25.5 \% \\
21\end{array}$ & $\begin{array}{c}23.1 \% \\
19\end{array}$ & $\begin{array}{c}20.0 \% \\
22\end{array}$ & $\begin{array}{c}18.23 \\
23\end{array}$ & $\begin{array}{c}21.9 \% \\
85\end{array}$ \\
\hline Alto & $\begin{array}{c}41.2 \% \\
16\end{array}$ & $\begin{array}{c}48.7 \% \\
11\end{array}$ & $\begin{array}{c}62.9 \% \\
4\end{array}$ & $\begin{array}{c}52.3 \% \\
13\end{array}$ & $\begin{array}{c}50.3 \% \\
44\end{array}$ \\
\hline Total & $\frac{31.4 \%}{51}$ & $\begin{array}{c}28.2 \% \\
39\end{array}$ & $\frac{11.4 \%}{35}$ & $\begin{array}{c}29.5 \% \\
44\end{array}$ & $\begin{array}{r}26.0 \% \\
169\end{array}$ \\
\hline & $100 \%$ & $100 \%$ & $100 \%$ & $100 \%$ & $100 \%$ \\
\hline
\end{tabular}

Morales-Guzmán (2020). La evaluación que practican los docentes universitarios.

En referencia a la tabla anterior, se pudo establecer que el 50.3\% de los docentes utilizan la evaluación para el aprendizaje en un nivel "medio", por lo tanto el nivel es aceptable, razón por la cual se acepta la hipótesis de investigación H3: La evaluación para el aprendizaje es aplicada por los docentes en un nivel "medio". Significa que la evaluación que practican 10 de 21 docentes que trabajan en la carrera de Profesorado de Enseñanza Media en Pedagogía y Técnico en Administración Educativa del CUNORI, está centrada en el proceso, porque, les interesan las cualidades de sus estudiantes y valoran lo cualitativo como lo cuantitativo. Los resultados son conforme a lo esperado y planteado.

\section{Discusión}

Se determina que la evaluación que practican los docentes durante los procesos de aprendizaje en la carrera de Profesorado de Enseñanza Media en Pedagogía y Técnico en Administración Educativa del CUNORI, posee un nivel de aplicación medio en sus tres manifestaciones: sumativa, formativa y para el aprendizaje. Estos datos indican que la variable evaluación docente se aplica en un 61.5\%. Particular atención merece el quinto semestre, en donde $77.1 \%$ ubica la evaluación docente en el nivel medio, siendo este semestre el de mayor porcentaje. 
Comparando estos resultados con la tesis doctoral de Lorenzana (2012) en donde concluye que la evaluación practicada por los docentes poco fomenta el aprendizaje, se observa una diferencia, pues en esta investigación, aunque sea en nivel medio, la evaluación contribuye para el aprendizaje de los estudiantes. De igual manera comparando los resultados con la tesis doctoral de Gómez (2017) en donde señala que los procesos evaluativos no deben centrarse en los logros cognitivos del estudiante, se evidencia que en los estudiantes de la carrera de Profesorado de Enseñanza Media en Pedagogía y Técnico en Administración Educativa del CUNORI, las evaluaciones son más integradoras, pues aprecian lo formativo y valoran el aprendizaje.

Se concluye que la evaluación que practican los docentes se está desarrollando en niveles aceptables, porque se aplica en un $61.5 \%$ en un nivel "medio", esto equivale a que, de 30 indicadores, 20 se encuentran arriba de la regularidad en su ejecución, Significa que de 21 docentes de la carrera de Profesorado de Enseñanza Media en Pedagogía y Técnico en Administración Educativa, 13 están aplicando pruebas objetivas y alternativas y se enfocan en la calificación como en el aprendizaje de sus estudiantes. Pero necesitan fortalecer las técnicas y estrategias cualitativas de evaluación y las evaluaciones de análisis y prácticas.

\section{Agradecimientos}

A la carrera de Pedagogía del Centro Universitario de Oriente por la apertura a la investigación realizada. Al coordinador Delfido Geovany Marroquín, docentes y estudiantes, por el apoyo durante el proceso de la investigación.

\section{Referencias}

Acuña Maldonado, Olía, y Ramírez Lugo, Francisco Ricardo (2010). Análisis del proceso de evaluación del aprendizaje en telesecundaria. Estudio de caso: Telesecundaria Federalizada \# 86. Ra Ximhai, 6(3), 421-443. doi: https://doi.org/10.35197/rx.06.03.2010.11.oa

Arias Lara, Sergio Alejandro, y de Arias Peñaloza, Milvia Lisette (2011). Evaluar los aprendizajes: un enfoque innovador. Educere, 15(51), 357-368.

Careaga, Adriana (2001). La evaluación como herramienta de transformación de la práctica docente. Educere, 5(15), 345-352.

Choc, F. (2014). Importancia de la evaluación formativa en los procesos educativos de docentes y estudiantes de la carrera de Profesorado en Pedagogía y Técnico en Administración Educativa del Centro Universitario de Chimaltenango. (Tesis de maestría). Universidad de San Carlos de Guatemala, Guatemala.

De la Orden, A. y Pimienta, J. H. (2016). Instrumento para determinar los tipos de evaluación utilizados por los profesores universitarios. Revista Electrónica de Investigación Educativa, 18(2), 40-52. 
Lorenzana, R. (2012). La evaluación de los aprendizajes basada en competencias en la enseñanza universitaria. (Tesis doctoral). Universidad Flensburg, Honduras.

Monteagudo, J. (2014). Las prácticas de evaluación en la materia de Historia de cuarto grado de ESO en la Comunidad Autónoma de la Región de Murcia. (Tesis doctoral). Universidad de Murcia, España.

Puentes, A. (2009). Tensiones y distensiones en la práctica evaluativa. Una propuesta de metaevaluación en el Programa Bachillerato Internacional. (Tesis de maestría). Pontificia Universidad Javeriana, Colombia.

Ruano, A. (2002). Evaluación Educativa Evaluar para aprender. Ciudad de Guatemala, Guatemala: DICADE, Ministerio de Educación.

Zambrano, A. (2014). Prácticas evaluativas para la mejora de la calidad del aprendizaje: Un estudio contextaulizado en La Unión Chile. Bellaterra. (Tesis doctoral). Universidad Autónoma de Barcelona, España.

\section{Sobre el autor}

\section{Rudy Rolando Morales Guzmán}

Profesor de Enseñanza Media en Ciencias Naturales con Orientación Ambiental, graduado de la Universidad de San Carlos en el año 2012. Es graduado de Licenciado en Pedagogía y Administración Educativa en la Universidad Panamericana en el año 2018, posteriormente se graduó en la Maestría en Docencia Universitaria con Orientación en Estrategias de Aprendizaje del Centro Universitario de Oriente. Es coautor del artículo científico Las actitudes humanas de los docentes en los procesos de aprendizaje en el año 2018.

Copyright (c) Rudy Rolando Morales Guzmán

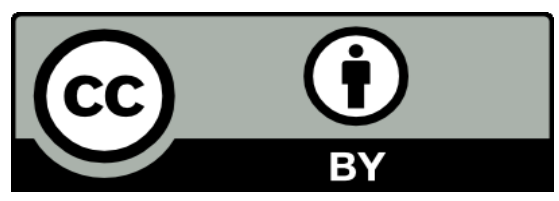

Este texto está protegido por una licencia CreativeCommons 4.0.

Usted es libre para compartir, copiar y redistribuir el material en cualquier medio o formato y adaptar el documento, remezclar, transformar y crear a partir del material para cualquier propósito, incluso comercialmente, siempre que cumpla la condición de atribución: usted debe reconocer el crédito de una obra de manera adecuada, proporcionar un enlace a la licencia, e indicar si se han realizado cambios. Puede hacerlo en cualquier forma razonable, pero no de forma tal que sugiera que tiene el apoyo del licenciante o lo recibe por el uso que hace. 